\title{
Miroslav Novotný
}

Ternary structures and groupoids

Czechoslovak Mathematical Journal, Vol. 41 (1991), No. 1, 90-98

Persistent URL: http://dml.cz/dmlcz/102437

\section{Terms of use:}

(C) Institute of Mathematics AS CR, 1991

Institute of Mathematics of the Czech Academy of Sciences provides access to digitized documents strictly for personal use. Each copy of any part of this document must contain these Terms of use.

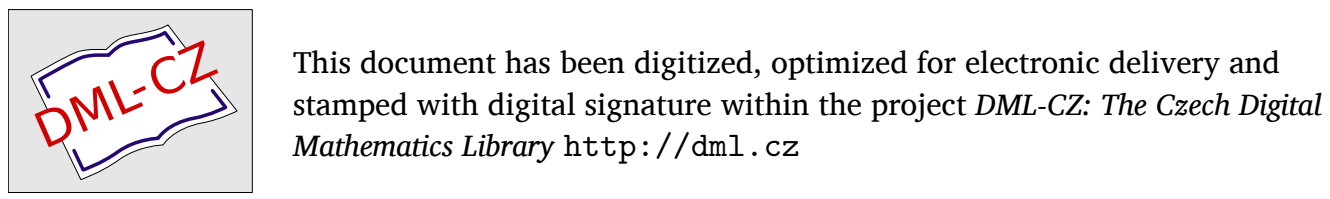




\title{
TERNARY STRUCTURES AND GROUPOIDS
}

\author{
Miroslav Novotný, Brno \\ (Received February 6, 1990)
}

\section{INTRODUCTION}

We prove that the category of ternary structures with strong homomorphisms as morphisms is isomorphic with a particular category of groupoids. Objects of the latter category are groupoids whose carriers are power sets and operations are totally additive. Morphisms of this category are totally additive and atom-preserving homomorphisms of those groupoids. By means of the isomorphism of these categories, the problem of constructing all strong homomorphisms between two ternary structures is reduced to the problem of constructing all totally additive and atom-preserving homomorphisms between two groupoids. The properties of a ternary structure influence the properties of the groupoid corresponding to the structure with respect to the above mentioned isomorphism and vice versa. The relationship between properties of a ternary structure and properties of the corresponding groupoid is also studied here.

\section{TOTALLY ADDITIVE AND ATOM-PRESERVING MAPPINGS}

For any set $A$ we denote by $\boldsymbol{P}(A)$ its power set, i.e., $\boldsymbol{P}(A)=\{X ; X \subseteq A\}$.

Let $A, A^{\prime}$ be sets.

A mapping $H$ of $\boldsymbol{P}(A)$ into $\boldsymbol{P}\left(A^{\prime}\right)$ is said to be totally additive if

$$
H\left(\bigcup\left\{X_{\iota} ; \iota \in I\right\}\right)=\bigcup\left\{H\left(X_{\iota}\right) ; \iota \in I\right\}
$$

for any system $\left\{X_{\iota} ; \iota \in I\right\}$ of subsets of the set $A$.

A mapping $H$ of $\boldsymbol{P}(A)$ into $\boldsymbol{P}\left(A^{\prime}\right)$ is referred to as atom-preserving if for any $x \in A$ there exists $x^{\prime} \in A^{\prime}$ such that $H(\{x\})=\left\{x^{\prime}\right\}$.

If $r$ is a relation from $A$ to $A^{\prime}$, then for any $X \in \boldsymbol{P}(A)$ we put $\mathbf{P}[r](X)=\left\{x^{\prime} \in A^{\prime}\right.$; there exists $x \in X$ with $\left.\left(x, x^{\prime}\right) \in \boldsymbol{r}\right\}$. Clearly, $\mathbf{P}[r]$ is a mapping of $\boldsymbol{P}(A)$ into $\boldsymbol{P}\left(A^{\prime}\right)$.

For any mapping $H$ of $\boldsymbol{P}(A)$ into $\boldsymbol{P}\left(A^{\prime}\right)$, we set $\mathbf{Q}[H]=\left\{\left(x, x^{\prime}\right) \in A \times A^{\prime} ; x^{\prime} \in\right.$ $\in H(\{x\})\}$. Clearly, $\mathbf{Q}[H]$ is a relation from $A$ to $A^{\prime}$.

In what follows, we use the following results of [6].

Lemma 1. If $H$ is a mapping of $\boldsymbol{P}(A)$ into $\boldsymbol{P}\left(A^{\prime}\right)$ and if $\mathbf{Q}[H]$ is a mapping, then 
for any $a \in A$ and any $a^{\prime} \in A^{\prime}$ the conditions $a^{\prime}=\mathbf{Q}[H](a),\left\{a^{\prime}\right\}=H(\{a\})$ are equivalent $([6]$ Corollary 1$)$.

Lemma 2. Let $A, A^{\prime}$ be sets, $H$ a mapping of $\boldsymbol{P}(A)$ into $\boldsymbol{P}\left(A^{\prime}\right)$. Then the following assertions are equivalent.

(i) $H$ is totally additive.

(ii) $H=(\mathbf{P} \circ \mathbf{Q})[H]([6]$ Lemma 4).

Lemma 3. Let $A, A^{\prime}$ be sets, $H$ a mapping of $\boldsymbol{P}(A)$ into $\boldsymbol{P}\left(A^{\prime}\right)$. Then the following assertions are equivalent.

(i) $H$ is totally additive and atom-preserving.

(ii) $\mathbf{Q}[H]$ is a mapping and $H=(\mathbf{P} \circ \mathbf{Q})[H]$ holds ([6] Corollary 2).

Lemma 4. If $A, A^{\prime}$ are sets and $h$ a mapping of $A$ into $A^{\prime}$, then $(\mathbf{Q} \circ \mathbf{P})[h]=h$ ([6] Lemma 5).

\section{CATEGORY TER}

The instruments of the theory of categories needed in what follows may be easily found in [3].

Let $A$ be a set and $t \subseteq A \times A \times A$. Then $t$ is said to be a ternary relation on $A$ and the ordered pair $(A, t)$ is called a ternary structure.

If $(A, t),\left(A^{\prime}, t^{\prime}\right)$ are ternary structures and $h$ is a mapping of $A$ into $A^{\prime}$, then $h$ is said to be a strong homomorphism of the ternary structure $(A, t)$ into $\left(A^{\prime}, t^{\prime}\right)$ whenever the following holds: For any $x \in A, y \in A, z^{\prime} \in A^{\prime}$, the condition $\left(h(x), z^{\prime}, h(y)\right) \in t^{\prime}$ is satisfied if and only if there exists $z \in A$ such that $(x, z, \mathrm{y}) \in t$, $h(z)=z^{\prime}$. (In [4] strong homomorphisms of ternary structures appear in a different meaning.)

Clearly, $1_{(A, t)}$ is a strong homomorphism of $(A, t)$ into $(A, t)$. We prove that the composite of two strong homomorphisms is a strong homomorphism, too. Let $h$ be a strong homomorphism of $(A, t)$ into $\left(A^{\prime}, t^{\prime}\right)$ and $k$ a strong homomorphism of $\left(A^{\prime}, t^{\prime}\right)$ into $\left(A^{\prime \prime}, t^{\prime \prime}\right)$; suppose that $x \in A, y \in A, z^{\prime \prime} \in A^{\prime \prime}$ are arbitrary.

If there exists $z \in A$ with $(x, z, y) \in t, z^{\prime \prime}=k(h(z))$, we set $h(z)=z^{\prime}$. Since $h$ is a strong homomorphism, we obtain $\left(h(x), z^{\prime}, h(y)\right) \in t^{\prime}$. As $k$ is a strong homomorphism and $k\left(z^{\prime}\right)=z^{\prime \prime}$, we have $\left(k(h(x)), z^{\prime \prime}, k(h(y))\right) \in t^{\prime \prime}$.

On the other hand, if $\left(k(h(x)), z^{\prime \prime}, k(h(y))\right) \in t^{\prime \prime}$, then the fact that $k$ is a strong homomorphism implies the existence of $z^{\prime} \in A^{\prime}$ with $\left(h(x), z^{\prime}, h(y)\right) \in t^{\prime}, z^{\prime \prime}=k\left(z^{\prime}\right)$, and the fact that $h$ is a strong homomorphism entails the existence of $z \in A$ with $(x, z, y) \in t, z^{\prime}=h(z)$. Thus $z^{\prime \prime}=k(h(z))$.

This proves the existence of a category TER whose objects are ternary structures and whose morphisms are strong homomorphisms of these structures.

Example 1. Let $(A, t),\left(A^{\prime}, t^{\prime}\right)$ be ternary structures. A mapping $h$ of $A$ into $A^{\prime}$ is said to be a homomorphism of $(A, t)$ into $\left(A^{\prime}, t^{\prime}\right)$ if $(x, z, y) \in t$ implies $(h(x), h(z)$. 
$h(y)) \in t^{\prime}$. Clearly, any strong homomorphism is a homomorphism but the converse need not hold as is seen from the following. Let $A, A^{\prime}$ be non-empty sets, $t=\emptyset$, $t^{\prime}=A^{\prime} \times A^{\prime} \times A^{\prime}$. If $h$ is an arbitrary mapping of $A$ into $A^{\prime}$, then $h$ is a homomorphism. For an arbitrary $a \in A$ we have $(h(a), h(a), h(a)) \in t^{\prime}$, but for any $z \in A$ with $h(z)=h(a)$ we obtain $(a, z, a) \notin t$. Thus, $h$ is no strong homomorphism of $(A, t)$ into $\left(A^{\prime}, t^{\prime}\right)$.

Example 2. Let $t$ be a binary operation on the set $A$, i.e., $t$ is a mapping of $A \times A$ into $A$. As usual, we denote by $t(a, b)$ the value assigned to the ordered pair $(a, b) \in$ $\in A \times A$. A set provided with a binary operation is said to be a groupoid (see e.g., [1], [2]). A groupoid with the carrier $A$ and with the operation $t$ will be denoted by $(A, t)$.

Let $(A, t),\left(A^{\prime}, t^{\prime}\right)$ be groupoids, $h$ a mapping of $A$ into $A^{\prime}$. Then $h$ is said to be a homomorphism of the groupoid $(A, t)$ into the groupoid $\left(A^{\prime}, t^{\prime}\right)$ if and only if $h(t(a, b))=t^{\prime}(h(a), h(b))$ holds for any $a, b$ in $A$.

A binary operation $t$ on a set $A$ may be considered to be a ternary relation: we put $(a, c, b) \in t^{\prime}$ if and only if $c=t(a, b)$. In what follows, we shall not distinguish between $t$ and $t^{\prime}$ and we shall write $t$ for $t^{\prime}$. Thus, a groupoid $(A, t)$ can be also regarded as a ternary structure. Therefore, if $(A, t),\left(A^{\prime}, t^{\prime}\right)$ are groupoids, we may consider groupoid-homomorphisms of $(A, t)$ into $\left(A^{\prime}, t^{\prime}\right)$ and strong homomorphisms of $(A, t)$ into $\left(A^{\prime}, t^{\prime}\right)$. We prove that groupoid-homomorphisms coincide with strong homomorphisms.

Let $(A, t),\left(A^{\prime}, t^{\prime}\right)$ be groupoids and $h$ a mapping of $A$ into $A^{\prime}$.

Suppose that $h$ is a groupoid-homomorphism and that $a, b \in A, c^{\prime} \in A^{\prime}$ are arbitrary. Then $\left(h(a), c^{\prime}, h(b)\right) \in t^{\prime}$ means $c^{\prime}=t^{\prime}(h(a), h(b))=h(t(a, b))$ which is equivalent to the existence of $c=t(a, b) \in A$ such that $h(c)=c^{\prime},(a, c, b) \in t$. Thus $h$ is a strong homomorphism of the ternary structure $(A, t)$ into $\left(A^{\prime}, t^{\prime}\right)$.

On the other hand, if $h$ is a strong homomorphism of the ternary structure $(A, t)$ into $\left(A^{\prime}, t^{\prime}\right)$ and if $a, b$ are arbitrary elements in $A$, then $c=t(a, b)$ exists and $(a, c, b) \in t$ holds. Put $c^{\prime}=h(c)$; then $\left(h(a), c^{\prime}, h(b)\right) \in t^{\prime}$ holds which means $h(c)=$ $=c^{\prime}=t^{\prime}(h(a), h(b))$, i.e., $h(t(a, b))=t^{\prime}(h(a), h(b))$. Thus, $h$ is a groupoid-homomorphism of $(A, t)$ into $\left(A^{\prime}, t^{\prime}\right)$.

Let $(A, t)$ be a ternary structure. The relation $t$ and the structure $(A, t)$ is said to be

(1) symmetric if and only if $(x, z, y) \in t$ implies $(y, z, x) \in t$ for any $x, y, z$ in $A$;

(2) asymmetric if and only if $(x, z, y) \in t$ implies $(y, z, x) \notin t$ for any $x, y, z$ in $A$;

(3) cyclic, if and only if $(x, z, y) \in t$ implies $(z, y, x) \in t$ for any $x, y, z$ in $A$;

(4) transitive if and only if $(x, z, y) \in t,(y, z, u) \in t$ imply $(x, z, u) \in t$ for any $x, y, z, u$ in $A$ (cf. [4], [5]).

Theorem 1. Let $(A, t),\left(A^{\prime}, t^{\prime}\right)$ be ternary structures and $h$ a strong homomorphism of $(A, t)$ onto $\left(A^{\prime}, t^{\prime}\right)$. If $(A, t)$ is symmetric (cyclic), then so is $\left(A^{\prime}, t^{\prime}\right)$.

Proof. Let us have $\left(x^{\prime}, z^{\prime}, y^{\prime}\right) \in t^{\prime}$. We take $x, y \in A$ such that $h(x)=x^{\prime}, h(y)=y^{\prime}$, 
which is possible because $h$ is surjective. Since $\left(h(x), z^{\prime}, h(y)\right) \in t^{\prime}$ and since $h$ is a strong homomorphism, there exists $z \in A$ such that $h(z)=z^{\prime}$ and $(x, z, y) \in t$. If $t$ is symmetric, then $(y, z, x) \in t$ which implies that $\left(y^{\prime}, z^{\prime}, x^{\prime}\right)=\left(h(y), z^{\prime}, h(x)\right) \in t^{\prime}$ and $t^{\prime}$ is symmetric. If $t$ is cyclic, then $(z, y, x) \in t$ which implies that $\left(z^{\prime}, y^{\prime}, x^{\prime}\right)=$ $=\left(h(z), y^{\prime}, h(x)\right) \in t^{\prime}$ and $t^{\prime}$ is cyclic.

Similar results for asymmetry and transitivity do not hold as is seen from the following.

Example 3. Suppose that $A=\{a, b, c, d\}, A^{\prime}=\left\{a^{\prime}, b^{\prime}, c^{\prime}\right\}, t=\{(a, b, c),(c, d, a)\}$, $t^{\prime}=\left\{\left(a^{\prime}, b^{\prime}, c^{\prime}\right),\left(c^{\prime}, b^{\prime}, a^{\prime}\right)\right\}$ and that $h(a)=a^{\prime}, h(b)=b^{\prime}=h(d), h(c)=c^{\prime}$. Then $(A, t)$ is asymmetric and transitive while $\left(A^{\prime}, t^{\prime}\right)$ is neither asymmetric nor transitive $\left(\left(a^{\prime}, b^{\prime}, c^{\prime}\right) \in t^{\prime},\left(c^{\prime}, b^{\prime}, a^{\prime}\right) \in t^{\prime}\right.$ would imply $\left(a^{\prime}, b^{\prime}, a^{\prime}\right) \in t^{\prime}$ for a transitive $\left.t^{\prime}\right)$. We prove that $h$ is a strong homomorphism of $(A, t)$ onto $\left(A^{\prime}, t^{\prime}\right)$. Indeed, if $(x, z, y) \in t$, then $(h(x), h(z), h(y)) \in t^{\prime}$. On the other hand, if $x \in A, y \in A, z^{\prime} \in A^{\prime}$ are such that $\left(h(x), z^{\prime}, h(y)\right) \in t^{\prime}$, then either $\left(h(x), z^{\prime}, h(y)\right)=\left(a^{\prime}, b^{\prime}, c^{\prime}\right)$ or $\left(h(x), z^{\prime}, h(y)\right)=$ $=\left(c^{\prime}, b^{\prime}, a^{\prime}\right)$. In the first case we have $x=a, y=c$ and choose $z=b$; in the latter we have $x=c, y=a$ and choose $z=d$. Thus $(x, z, y) \in t, h(z)=z^{\prime}$.

\section{TOTALLY ADDITIVE OPERATIONS}

Let $(A, t)$ be a ternary structure. For any $X \in \boldsymbol{P}(A), Y \in \boldsymbol{P}(A)$ we set

$$
\begin{aligned}
& \mathbf{R}[t](X, Y)= \\
& =\{z \in A ; \text { there exist } x \in X \text { and } y \in Y \text { such that }(x, z, y) \in t\} .
\end{aligned}
$$

Clearly, $(\boldsymbol{P}(A), \mathbf{R}[t])$ is a groupoid.

Theorem 2. Let $(A, t),\left(A^{\prime}, t^{\prime}\right)$ be ternary structures and $h$ a mapping of $A$ into $A^{\prime}$. Then the following assertions are equivalent.

(i) $h$ is a strong homomorphism of $(A, t)$ into $\left(A^{\prime}, t^{\prime}\right)$.

(ii) $\mathbf{P}[h]$ is a totally additive atom-preserving homomorphism of $(\boldsymbol{P}(A), \mathbf{R}[t])$ into $\left(\boldsymbol{P}\left(A^{\prime}\right), \mathbf{R}\left[t^{\prime}\right]\right)$.

Proof. If $(i)$ holds and $X, Y \in P(A)$ are arbitrary, then $\mathbf{P}[h](\mathbf{R}[t](X, Y))=$ $=\mathbf{P}[h](\{z \in A$; there exist $x \in X, y \in Y$ with $(x, z, y) \in t\})=\{h(z)$; there exist $x \in X, y \in Y$ with $(x, z, y) \in t\}$. Similarly, $\mathbf{R}\left[t^{\prime}\right](\mathbf{P}[h](X), \mathbf{P}[h](Y))=\mathbf{R}\left[t^{\prime}\right](\{h(x)$; $x \in X\},\{h(y) ; y \in Y\})=\left\{z^{\prime} \in A^{\prime} ;\right.$ there exist $x \in X, y \in Y$ with $\left.\left(h(x), z^{\prime}, h(y)\right) \in t^{\prime}\right\}$. The fact that $h$ is a strong homomorphism implies that $\mathbf{P}[h](\mathbf{R}[t](X, Y))=$ $=\mathbf{R}\left[t^{\prime}\right](\mathbf{P}[h](X), \mathbf{P}[h](Y))$ for any $X \in \boldsymbol{P}(A)$ and any $Y \in \boldsymbol{P}(A)$ which means that $\mathbf{P}[h]$ is a homomorphism of the groupoid $(\boldsymbol{P}(A), \mathbf{R}[t])$ into $\left(\boldsymbol{P}\left(A^{\prime}\right), \mathbf{R}\left[t^{\prime}\right]\right)$.

By definition, $\mathbf{P}[h]$ is totally additive. By Lemma 4 , we have $(\mathbf{Q} \circ \mathbf{P})[h]=h$. Thus, $\mathbf{Q}[\mathbf{P}[h]]$ is a mapping and $(\mathbf{P} \circ \mathbf{Q})[\mathbf{P}[h]]=(\mathbf{P} \circ \mathbf{Q} \circ \mathbf{P})[h]=\mathbf{P}[h]$ and, therefore, $\mathbf{P}[h]$ is totally additive and atom preserving by Lemma 3 . Thus $(i i)$ holds.

Suppose that (ii) is satisfied. If $\left(h(x), z^{\prime}, h(y)\right) \in t^{\prime}$ holds for $x \in A, y \in A, z^{\prime} \in A^{\prime}$, 
then $z^{\prime} \in \mathbf{R}\left[t^{\prime}\right](\{h(x)\},\{h(y)\})=\mathbf{R}\left[t^{\prime}\right](\mathbf{P}[h](\{x\}), \mathbf{P}[h](\{y\}))=$ $=\mathbf{P}[h](\mathbf{R}[t](\{x\},\{y\}))=\mathbf{P}[h](\{z ;(x, z, y) \in t\})=\{h(z) ;(x, z, y) \in t\}$ which implies the existence of $z \in A$ with $(x, z, y) \in t, h(z)=z^{\prime}$. On the other hand, if $z \in A,(x, z, y) \in t, h(z)=z^{\prime}$. hold, then $z \in \mathbf{R}[t](\{x\},\{y\})$ and, therefore, $z^{\prime} \in$ $\in \mathbf{P}[h]\left(\mathbf{R}[t](\{x\},\{y\})=\mathbf{R}\left[t^{\prime}\right](\mathbf{P}[h](\{x\}), \quad \mathbf{P}[h](\{y\}))=\mathbf{R}\left[t^{\prime}\right](\{h(x)\}, \quad\{h(y)\})\right.$, which means $\left(h(x), z^{\prime}, h(y)\right) \in t^{\prime}$. Hence, $h$ is a strong homomorphism and $(i)$ holds.

Let $A$ be a set, $N$ a binary operation on $\boldsymbol{P}(A)$. The operation $N$ is said to be totally additive if the following conditions are satisfied.

$\left(t a_{1}\right)$ For any system $\left\{X_{\iota} ; \iota \in I\right\}$ of subsets of $A$ and for any subset $Y$ of $A$ the equation $N\left(\bigcup\left\{X_{\iota} ; \iota \in I\right\}, Y\right)=\bigcup\left\{N\left(X_{\iota}, Y\right) ; \iota \in I\right\}$ holds.

$\left(t a_{2}\right)$ For any system $\left\{Y_{x} ; \varkappa \in K\right\}$ of subsets of $A$ and for any subset $X$ of $A$ the equation $N\left(X, \cup\left\{Y_{\varkappa} ; \varkappa \in K\right\}\right)=\bigcup\left\{N\left(X, Y_{\varkappa} ;\right) ; \varkappa \in K\right\}$ holds.

Lemma 5. If $(A, t)$ is a ternary structure, then $\mathbf{R}[t]$ is a totally additive operation.

Proof. If $\left\{X_{\iota} ; \iota \in I\right\}$ is a system of subsets of $A$ and $Y$ is a subset of $A$, then $\mathbf{R}[t]\left(\bigcup\left\{X_{\iota} ; \iota \in I\right\}, Y\right)=\left\{z \in A\right.$; there exist $x \in \bigcup\left\{X_{\iota} ; \iota \in I\right\}$ and $y \in Y$ with $(x, z, y) \in$ $\in t\}=\left\{z \in A\right.$; there exist $\iota \in I, x \in X_{i}, y \in Y$ with $\left.(x, z, y) \in t\right\}=\bigcup\{\{z \in A$; there exist $x \in X_{i}, y \in Y$ with $\left.\left.(x, z, y) \in t\right\}, \iota \in I\right\}=\bigcup\left\{R\left(X_{\iota}, Y\right) ; \iota \in I\right\}$, which is $\left(t a_{1}\right)$. Similarly, we prove that $\left(t a_{2}\right)$ holds.

If $A$ is a set and $N$ a binary operation on $\boldsymbol{P}(A)$, then we put

$$
\mathbf{S}[N]=\{(x, z, y) \in A \times A \times A ; z \in N(\{x\},\{y\})\} .
$$

Lemma 6. For any ternary structure $(A, t)$ the condition $(\mathbf{S} \circ \mathbf{R})[t]=t$ holds.

Proof. Clearly, $(x, z, y) \in t$ is equivalent to $z \in \mathbf{R}[t](\{x\},\{y\})$, which means $(x, z, y) \in \mathbf{S}[\mathbf{R}[t]]$. Thus, $t=\mathbf{S}[\mathbf{R}[t]]=(\mathbf{S} \circ \mathbf{R})[t]$.

Lemma 7. If $A$ is a set and $N$ a totally additive operation on $\boldsymbol{P}(A)$, then $N(X, Y)=$ $=\bigcup\{N(\{x\},\{y\}) ;(x, y) \in X \times Y\}$ holds for arbitrary $X \in \boldsymbol{P}(A)$ and $Y \in \boldsymbol{P}(A)$.

Proof. $N(X, Y)=N(\bigcup\{\{x\} ; x \in X\}, Y)=\bigcup\{N(\{x\}, Y\} ; x \in X\}=\bigcup\{N(\{x\}$, $\bigcup\{\{y\} ; y \in Y\}) ; \quad x \in X\}=\bigcup\{\bigcup\{N(\{x\},\{y\}) ; y \in Y\}, x \in X\}=\bigcup\{N(\{x\},\{y\}) ;$ $(x, y) \in X \times Y\}$.

Lemma 8. If $A$ is a set and $N$ a totally additive operation on $\boldsymbol{P}(A)$, then $N=$ $=(\mathbf{R} \circ \mathbf{S})[N]$.

Proof. For arbitrary $X \in \boldsymbol{P}(A), Y \in \boldsymbol{P}(A)$ the condition $z \in N(X, Y)$ is equivalent to the existence of $(x, y) \in X \times Y$ such that $z \in N(\{x\},\{y\})$ by Lemma 7. By definition of $\mathbf{S}$, this means $(x, z, y) \in \mathbf{S}[N]$ for some $x \in X$ and some $y \in Y$ which may be written as $z \in \mathbf{R}[\mathbf{S}[N]](X, Y)$. Thus, we have $N=\mathbf{R}[\mathbf{S}[N]]=(\mathbf{R} \circ \mathbf{S})[N]$.

Theorem 3. Let $A, A^{\prime}$ be sets $(\boldsymbol{P}(A), N),\left(\boldsymbol{P}\left(A^{\prime}\right), N^{\prime}\right)$ groupoids with totally additive operations, $H$ a totally additive mapping of $\boldsymbol{P}(A)$ into $\boldsymbol{P}\left(A^{\prime}\right)$. Then the following conditions are equivalent. 
(i) $\mathbf{Q}[H]$ is a strong homomorphism of the ternary structure $(A, \mathbf{S}[N])$ into $\left(A^{\prime}, \mathbf{S}\left[N^{\prime}\right]\right)$.

(ii) $H$ is a totally additive atom-preserving homomorphism of the groupoid $(\boldsymbol{P}(A), N)$ into $\left(\boldsymbol{P}\left(A^{\prime}\right), N^{\prime}\right)$.

Proof. Put $h=\mathbf{Q}[H], t=\mathbf{S}[N], t^{\prime}=\mathbf{S}\left[N^{\prime}\right]$. We obtain $\mathbf{P}[h]=H$ by Lemma 2 , $\mathbf{R}[t]=N, \mathbf{R}\left[t^{\prime}\right]=N^{\prime}$ by Lemma 8 . Then (i) coincides with $(i)$ of Theorem 2 and $(i i)$ is identical with $(\mathrm{ii})$ of the same theorem, i.e., they are equivalent.

\section{CATEGORY PGR}

We now introduce the category PGR (of Power-set GRoupoids). Objecis of this category are groupoids of the form $(\boldsymbol{P}(A), N)$ where $A$ is a set and $N$ is a totally additive operation on $\boldsymbol{P}(A)$. By a morphism of the object $(\boldsymbol{P}(A), N)$ into the object $\left(\boldsymbol{P}\left(A^{\prime}\right), N^{\prime}\right)$ in $\mathbf{P G R}$ we mean a totally additive atom-preserving homomorphism of the groupoid $(\boldsymbol{P}(A), N)$ into $\left(\boldsymbol{P}\left(A^{\prime}\right), N^{\prime}\right)$.

Since $1_{(\boldsymbol{P}(A), N)}$ is a totally additive atom-preserving homomorphism of $(\boldsymbol{P}(A), N)$ into itself and since the composite of two totally additive atom-preserving homomorphisms is a totally additive atom-preserving homomorphism, PGR is a category.

\section{ISOMORPHISM OF CATEGORIES TER AND PGR}

We now introduce two înctors. $F$ is a functor of the category TER into PGR and $G$ is a functor of the category $\mathbf{P G R}$ into TER. These functors will be defined by presenting object mappings $F o$, Go and morphism mappings $F m, G m$.

If $(A, t)$ is an object in the category TER and $h$ a morphism in this category, we put

$$
F o(A, t)=(\boldsymbol{P}(A), \mathbf{R}[t]), \quad F m(h)=\mathbf{P}[h] .
$$

If $(\boldsymbol{P}(A), N)$ is an object in $\mathbf{P G} \mathbf{R}$ and $H$ is a morphism in this category, we put

$$
G o(\boldsymbol{P}(A), N)=(A, \mathbf{S}[N]), \quad G m(H)=\mathbf{Q}[H] .
$$

Main Theorem. $F$ is a functor of the category TER into $\mathbf{P G R}$ and $G$ is a functor of the category PGR into TER such that both $F \circ G$ and $G \circ F$ are identity functors.

Proof. (1) By Lemma 5, Fo $(A, t)$ is an object in $\mathbf{P G R}$ for any object $(A, t)$ in TER. Furthermore, $F m(h)$ is a morphism in $\mathbf{P G R}$ for any morphism $h$ in TER by Theorem 2. Clearly, $F m\left(1_{(A, t)}\right)=1_{F o(A, t)}$ for any object $(A, t)$ in TER. Finally, if $h$ is a morphism of $(A, t)$ into $\left(A^{\prime}, t^{\prime}\right)$ in TER and $h^{\prime}$ is a morphism of $\left(A^{\prime}, t^{\prime}\right)$ into $\left(A^{\prime \prime}, t^{\prime \prime}\right)$ in TER, we obiain $\left(F m\left(h^{\prime}\right) \circ F m(h)\right)(X)=\left\{\left(h^{\prime} \circ h\right)(x) ; x \in X\right\}=\left(F m\left(h^{\prime} \circ h\right)\right)(X)$ for any $X \in \boldsymbol{P}(A)$, which means $F m\left(h^{\prime}\right) \circ F m(h)=F m\left(h^{\prime} \circ h\right)$.

This implies that $F$ is a functor.

(2) Similarly, $G o(\boldsymbol{P}(A), N)=(A, \mathbf{S}[N])$ is an object in TER for any object $(\boldsymbol{P}(A), N)$ in $\mathbf{P G R}$. Furthermore, $G m(H)$ is a morphism in TER for any morphism $H$ 
in PGR by Theorem 3. Clearly, $\operatorname{Gm}\left(1_{(\boldsymbol{P}(\boldsymbol{A}), N)}\right)=\left\{(x, y) \in A \times A ; y \in 1_{(\boldsymbol{P}(\boldsymbol{A}), N)}(\{x\})\right\}=$ $=1_{A}=1_{G o(P(A), N)}$.

If $H$ is a morphism of $(\boldsymbol{P}(A), N)$ into $\left(\boldsymbol{P}\left(A^{\prime}\right), N^{\prime}\right)$ in $\mathbf{P G} \mathbf{R}$ and $H^{\prime}$ is a morphism of $\left(\boldsymbol{P}\left(A^{\prime}\right), N^{\prime}\right)$ into $\left(\boldsymbol{P}\left(A^{\prime \prime}\right), N^{\prime \prime}\right)$ in $\mathbf{P G R}$, then $\mathbf{Q}[H], \mathbf{Q}\left[H^{\prime}\right], \mathbf{Q}\left[H^{\prime} \circ H\right]$ are morphisms in TER, i.e., mappings. Let $x \in A, x^{\prime \prime} \in A^{\prime \prime}$ be arbitrary. By Lemma 1 , the condition $x^{\prime \prime}=\mathbf{Q}\left[H^{\prime} \circ H\right](x)$ is equivalent to $\left\{x^{\prime \prime}\right\}=H^{\prime}(H(\{x\}))$. Since $H, H^{\prime}$ are atompreserving, we obtain $H(\{x\})=\left\{x^{\prime}\right\},\left\{x^{\prime \prime}\right\}=H^{\prime}\left(\left\{x^{\prime}\right\}\right)$ for some $x^{\prime} \in A^{\prime}$. By Lemma 1 , we obtain $x^{\prime}=\mathbf{Q}[H](x), x^{\prime \prime}=\mathbf{Q}\left[H^{\prime}\right]\left(x^{\prime}\right)$, i.e. $x^{\prime \prime}=\left(\mathbf{Q}\left[H^{\prime}\right] \circ \mathbf{Q}[H]\right)(x)$, which means $\mathbf{Q}\left[H^{\prime} \circ H\right]=\mathbf{Q}\left[H^{\prime}\right] \circ \mathbf{Q}[H]$, i.e. $G m\left(H^{\prime} \circ H\right)=G m\left(H^{\prime}\right) \circ G m(H)$.

It follows that $G$ is a functor.

(3) If $(A, t)$ is an object in TER, then $F o(A, t)=(\boldsymbol{P}(A), \mathbf{R}[t])$ and $G o(F o(A, t))=$ $=(A, \mathbf{S}[\mathbf{R}[t]])=(A, t)$ by Lemma 6. Similarly, if $(\boldsymbol{P}(A), N)$ is an object in PGR, we obtain $\operatorname{Go}(\boldsymbol{P}(A), N)=(A, \mathbf{S}[N])$ and $F o(G o(\boldsymbol{P}(A), N))=(\boldsymbol{P}(A), \mathbf{R}[\mathbf{S}[N]])=$ $=(\boldsymbol{P}(A), N)$ by Lemma 8 . Thus, $G o \circ F o$ is the identity on the class of all objects in TER and $F o \circ G o$ is the identity on the class of all objects in PGR.

If $h$ is a morphism in TER, we have $F m(h)=\mathbf{P}[h]$ and $\operatorname{Gm}(F m(h))=\mathbf{Q}[\mathbf{P}[h]]=$ $=h$ by Lemma 4. Finally, if $H$ is a morphism in PGR, we obtain $G m(H)=\mathbf{Q}[H]$, $F m(G m(H))=\mathbf{P}[\mathbf{Q}[H]]=H$ by Lemma 2. Hence, $G m \circ F m$ is the identity on the class of all morphisms in TER and $F m \circ G m$ is the identity on the class of all morphisms in PGR.

We have proved that $F \circ G$ and $G \circ F$ are identity functors.

Corollary 1. The functor $F$ is an isomorphism of the category TER onto PGR and the functor $G$ is an isomorphism of the category PGR onto TER.

Corollary 2. Let $(A, t),\left(A^{\prime}, t^{\prime}\right)$ be ternary structures.

(i) For any strong homomorphism $h$ of the structure $(A, t)$ into $\left(A^{\prime}, t^{\prime}\right)$ there exists a totally additive atom-preserving homomorphism $H$ of the groupoid $(\boldsymbol{P}(A), \mathbf{R}[t])$ into $\left(\boldsymbol{P}\left(A^{\prime}\right), \mathbf{R}\left[t^{\prime}\right]\right)$ such that $h=\mathbf{Q}[H]$.

(ii) If $H$ is an arbitrary totally additive atom-preserving homomorphism of the groupoid $(\boldsymbol{P}(A), \mathbf{R}[t])$ into $\left(\boldsymbol{P}\left(A^{\prime}\right), \mathbf{R}\left[t^{\prime}\right]\right)$, then $\mathbf{Q}[H]$ is a strong homomorphism of the structure $(A, t)$ into $\left(A^{\prime}, t^{\prime}\right)$.

\section{POWER SET GROUPOIDS OF PARTICULAR STRUCTURES}

We now investigate the question whether a particular property (symmetry, asymmetry, cyclicity, transitivity) of a ternary structure $(A, t)$ influences its power set groupoid $(\boldsymbol{P}(A), \mathbf{R}[t])$.

Theorem 4. Let $(A, t)$ be a ternary structure. Then the following assertions are equivalent.

(i) $(A, t)$ is symmetric.

(ii) For any $X, Y \in \boldsymbol{P}(A)$ the condition $\mathbf{R}[t](X, Y)=\mathbf{R}[t](Y, X)$ holds. 
Proof. Suppose that $(i)$ holds and that $z \in \mathbf{R}[t](X, Y)$ is arbitrary. Then there exist $x \in X$ and $y \in Y$ such that $(x, z, y) \in t$ which implies that $(y, z, x) \in t$ and, therefore, $z \in \mathbf{R}[t](Y, X)$ holds. Thus, $\mathbf{R}[t](X, Y) \subseteq \mathbf{R}[t](Y, X)$ which implies $(i i)$.

If $(i i)$ holds and $(x, z, y) \in t$ it satisfied, then $z \in \mathbf{R}[t](\{x\},\{y\})=\mathbf{R}[t](\{y\},\{x\})$ which implies that $(y, z, x) \in t$. We have proved $(i)$.

Theorem 5. Let $(A, t)$ be a ternary structure. Then the following assertions are equivalent.

(i) $(A, t)$ is transitive.

(ii) For any $X \in \boldsymbol{P}(A), U \in \boldsymbol{P}(A), z \in A$ the condition $\mathbf{R}[t](X,\{z\}) \cap$ $\cap \mathbf{R}[t](\{z\}, U) \subseteq \mathbf{R}[t](X, U)$ is satisfied.

Proof. If $(i)$ holds and $X \in \boldsymbol{P}(A), U \in \boldsymbol{P}(A), z \in A$ are arbitrary, then for any $y \in \mathbf{R}[t](X,\{z\}) \cap \mathbf{R}[t](\{z\}, U)$ there are $x \in X$ and $u \in U$ such that $(x, y, z) \in t$, $(z, y, u) \in t$. The transitivity of $t$ implies that $(x, y, u) \in t$, which means $y \in \mathbf{R}[t](X, U)$. Thus, (ii) holds.

If (ii) holds, then, in particular, for any $x, y, z, u$ in $A$ the condition $(x, z, y) \in t$, $(y, z, u) \in t$ means $z \in \mathbf{R}[t](\{x\},\{y\}) \cap \mathbf{R}[t](\{y\},\{u\})$. By (ii), we obtain $z \in$ $\in \mathbf{R}[t](\{x\},\{u\})$, i.e. $(x, z, u) \in t$. Thus, $(i)$ holds.

The last result provokes the question of the characteristic property of $(A, t)$ such that $\mathbf{R}[t](X, Z) \cap \mathbf{R}[t](Z, U) \subseteq \mathbf{R}[t](X, U)$ for any $X, Z, U$ in $\boldsymbol{P}(A)$.

The structure $(A, t)$ and the ternary relation $t$ will be said to be storngly transitive if for any $x, y, z, u, v$ in $A$ the following condition is satisfied: $(x, z, y) \in t,(v, z, u) \in t$ imply $(x, z, u) \in t$.

Theorem 6. Let $(A, t)$ be a ternary structure. Then the following assertions are equivalent.

(i) $(A, t)$ is strongly transitive.

(ii) For any $X, Z, U$ in $\boldsymbol{P}(A)$ the condition $\mathbf{R}[t](X, Z) \cap \mathbf{R}[t](Z, U) \subseteq$ $\subseteq \mathbf{R}[t](X, U)$ is satisfied.

Proof. If $(i)$ holds and $X, Z, U$ in $\boldsymbol{P}(A)$ are arbitrary, then for any $y \in \mathbf{R}[t](X, Z) \cap$ $\cap \mathbf{R}[t](Z, U)$ there exist $x \in X, z_{1} \in Z, z_{2} \in Z$, and $u \in U$ such that $\left(x, y, z_{1}\right) \in t$, $\left(z_{2}, y, u\right) \in t$. Since $t$ is strongly transitive, we obtain $(x, y, u) \in t$ which implies that $y \in \mathbf{R}[t](X, U)$. Thus, (ii) holds.

If $(i i)$ holds and $(x, z, y) \in t,(v, z, u) \in t$ are satisfied, then $z \in \mathbf{R}[t](\{x\},\{y, v\}) \cap$ $\cap \mathbf{R}[t](\{y, v\},\{u\}) \subseteq \mathbf{R}[t](\{x\},\{u\})$ and, thus, $(x, z, u) \in t$ which means that $(i)$ holds.

Theorem 7. Let $(A, t)$ be a ternary structure. Then the following assertions are equivalent.

(i) $(A, t)$ is cyclic.

(ii) For any $x, y, z$ in $A$ the following condition is satisfied: $z \in \mathbf{R}[t](\{x\},\{y\})$ if and only if $y \in \mathbf{R}[t](\{z\},\{x\})$. 
Proof. If $(i)$ holds and $z \in \mathbf{R}[t](\{x\},\{y\})$, then $(x, z, y) \in t$ which implies that $(z, y, x) \in t$ and, thus, $y \in \mathbf{R}[t](\{z\},\{x\})$. Similarly, $y \in \mathbf{R}[t](\{z\},\{x\})$ implies $z \in \mathbf{R}[t](\{x\},\{y\})$. Thus $(i i)$ holds.

If (ii) holds and $(x, z, y) \in t$, then $z \in \mathbf{R}[t](\{x\},\{y\})$ which implies that $y \in$ $\in \mathbf{R}[t](\{z\},\{x\})$. Hence $(z, y, x) \in t$ and $(i)$ holds.

Theorem 8. Let $(A, t)$ be a ternary structure. Then the following assertions are equivalent.

(i) $(A, t)$ is asymmetric.

(ii) For any $x, y$ in $A$ the following condition is satisfied: $\mathbf{R}[t](\{x\},\{y\}) \cap$ $\cap \mathbf{R}[t](\{y\},\{x\})=\emptyset$.

Proof. If $(i)$ holds and $z \in \mathbf{R}[t](\{x\},\{y\}) \cap \mathbf{R}[t](\{y\},\{x\})$, then $(x, z, y) \in t$, $(y, z, x) \in t$ which is a contradiction. Hence, $(i)$ implies $(i i)$.

If $(i i)$ holds and $(x, z, y) \in t$, then $z \in \mathbf{R}[t](\{x\},\{y\})$ which implies that $z \notin$ $\notin \mathbf{R}[t](\{y\},\{x\})$ and, therefore, $(y, z, x) \notin t$. Thus, (ii) implies $(i)$.

\section{References}

[1] O. Borůvka: Úvod do teorie grup. (Introduction to group theory.) Královská česká společnost nauk, Praha 1944.

[2] O. Borüvka: Grundlagen der Gruppoid- und Gruppentheorie. VEB Deutscher Verlag der Wissenschaften, Berlin 1960.

[3] S. Mac Lane: Categories for the working mathematician. Springer, New York-HeidelbergBerlin 1971.

[4] V. Novák, M. Novotný: Universal cyclically ordered sets. Czechoslovak Math. J. 35 (110) (1985), 158-161.

[5] V. Novák, M. Novotný: Transitive ternary relations and quasiorderings. Archivum Math. Brno 25 (1989), 5- 12.

[6] M. Novotny: Construction of all strong homomorphisms of binary structures. Submitted to Czechoslovak Math. J.

Author's address: 66295 Brno, Janáčkovo nám. 2a, Czechoslovakia (PF MU). 